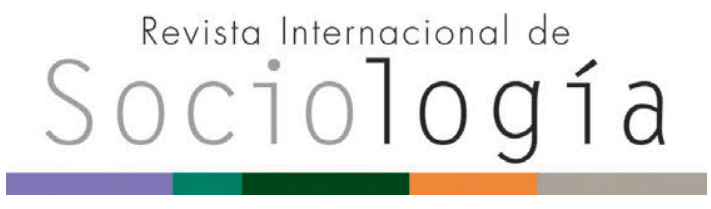

Revista Internacional de Sociología RIS

vol. 75 (1), e056, enero-marzo, 2017, ISSN-L:0034-9712

doi: http://dx.doi.org/10.3989/ris.2017.75.1.16.183

\title{
TIEMPO Y DESIGUALDAD EN LAS DINÁMICAS LABORALES Y FAMILIARES
}

\section{TIME AND INEQUALITY IN LABOR AND FAMILY DYNAMICS}

\author{
TOMÁS CANO \\ Universidad Autónoma de Barcelona y Universidad Pompeu Fabra \\ tomasc1010@gmail.com \\ ORCID iD: http://orcid.org/0000-0002-1804-164X
}

Cómo citar este artículo / Citation: Cano, T. 2017. "Tiempo y desigualdad en las dinámicas laborales y familiares". Revista Internacional de Sociología 75 (1): e056. doi: http:// dx.doi.org/10.3989/ris.2017.75.1.16.183

Copyright: (C) 2017 CSIC. Este es un artículo de acceso abierto distribuido bajo los términos de la licencia Creative Commons Attribution (CC BY) España 3.0.

Recibido: 06/10/2016. Aceptado: 12/12/2016.

Publicado on line: 07/03/2017

\section{Resumen}

Los cambios sociales experimentados en las últimas décadas han modificado los usos del tiempo de los individuos. Sin embargo, cómo se emplea el tiempo viene condicionado por la posición que se ocupa en la estructura social. Este trabajo argumenta que la desigualdad entre los distintos estatus socioeconómicos en el poder sobre el uso del tiempo está aumentando. Mientras tanto, las políticas sociales fallan en su potencial igualador. Para ello este ensayo sintetiza críticamente tres libros de reciente publicación centrados en los casos americano, francés y español. Tres elementos que forman la organización social del tiempo son discutidos: (a) la dependencia en el control sobre el tiempo entre grupos sociales y la endogeneidad entre el tiempo familiar y laboral; (b) la ambivalencia de la flexibilidad en función de su vinculación con el género y el estatus socioeconómico; y (c) la necesidad de una reevaluación de las políticas sociales destinadas a proteger el tiempo familiar de los trabajadores.

\section{Palabras Clave}

Conciliación laboral y familiar; Empleo del tiempo; Flexibilidad; Género; Política social.

\section{Abstract}

The social changes in last decades have modified the time use of individuals. However, how time is used varies according to the position occupied within the social structure. This paper argues that the social inequality in the power over the time use is increasing among different socioeconomic statuses, while social policies are failing in their equalizer potential. To do so, this essay critically synthetizes three recent published books covering the cases of United States, France and Spain. Three elements that shape the social organization of time are being discussed: (a) the dependency among different social groups in time's control and the endogeneity between family time and work time; (b) the ambivalence of flexibility regarding its relation with gender and socioeconomic status; and (c) the need for a new assessment of the social policies designated to protect the workers' family time.

\section{KeYWORDS}

Flexibility; Gender; Social policy; Time use; WorkFamily Balance. 


\section{INTRODUCCIÓN ${ }^{1}$}

"No tengo tiempo..." es una de las frases que más escucharemos a lo largo de nuestra vida. No obstante, el tiempo ni se tiene ni se deja de tener: nadie tiene (el) tiempo. El tiempo podría definirse como una secuencia continuada de prácticas. El tiempo social se implanta en la era de la industrialización con objeto de medir de manera exacta los contornos de la organización social: sin la estandarización de la medida temporal no podía haber transacciones financieras, elemento base del capitalismo. Es así como el tiempo social marcado por el reloj termina imponiéndose al tiempo biológico, marcado por las pautas corporales del ciclo vital y la naturaleza (Castell, 2009). Este sería el origen de un tiempo disciplinario, que impone los ritmos de organización social sobre el tiempo biológico.

El tiempo es a su vez un recurso inmaterial. Es el único recurso del que todos los individuos disponen exactamente de la misma cantidad diaria: 24 horas. Así, el tiempo es el único recurso que los individuos en situaciones desaventajadas pueden tener gratuitamente. Sin embargo, la forma en la que las personas usan y controlan su tiempo depende de su posición social. Por lo tanto, el tiempo puede ser estudiado de manera empírica para revelar su relación con las estructuras de poder y desigualdad.

Esto es lo que analizan los libros de Dan Clawson y Naomi Gerstel Unequal Time: Gender, Class and Family Employment Schedules; Martín Criado y Carlos Prieto Conflictos por el tiempo: Poder, relación salarial y relaciones de género y Kathleen Gerson The Unfinished Revolution: Coming of Age in a New Era of Gender, Work and Family, este último más exclusivamente centrado en las relaciones de género. Este conjunto de recientes publicaciones vuelve a poner con fuerza sobre el tapete la cuestión del equilibrio temporal en las dos principales esferas de la vida: el empleo y la familia.

Conflictos por el Tiempo: Poder, Relación Salarial y Relaciones de Género es una compilación de trabajos coordinada por Enrique Martín Criado y Carlos Prieto. El libro comienza con una introducción teórica de los editores, sentando las bases de algunos de los principales conceptos que articularán los posteriores capítulos: del origen del análisis de las disputas temporales ya teorizadas por Marx, al impacto producido por los recientes cambios de género en el uso del tiempo. A continuación el primer capítulo empírico del libro, de Martínez García, se centra en la flexibilidad laboral contemporánea como parte de una economía de la disponibilidad temporal. Centrándose en tres casos -enfermeras, sector bancario y operarios de limpieza-, el autor analiza la desarticulación de las estructuras temporales y las implicaciones de dicha desarticulación sobre las disponibilidades temporales de los em- pleados frente a sus superiores. En el capítulo dos, Carvajal Soria y Martín Criado analizan las batallas por el tiempo por parte de trabajadores, sindicatos y empleadores en las grandes superficies comerciales españolas desde 1976 a 2011. Para ello se sirven de entrevistas en profundidad, documentos legales y sentencias judiciales. Estos autores concluyen que el Estado, a través de la legislación, contiene el poder suficiente y necesario para frenar y mediar sobre las lógicas de imposición de estructuras temporales imposibles por parte del mercado a los empleados. El capítulo tres, de Thébaud-Mony, se centra en la gestión del tiempo en la industria nuclear francesa. Ésta autora focaliza la individualización extrema de las trayectorias profesionales, concluyendo cómo los trabajadores del sector nuclear, bajo imposiciones de duras formas de represión y temor, terminan por no beneficiarse de las garantías sindicales. El capítulo cuatro, de carácter muy breve y también centrado en Francia, lo escribe Hélène Bretin. La autora saca a la luz las relaciones de dominación y división sexual del trabajo en el sector de las trabajadoras de limpieza. Paul Bouffartigue abre la segunda parte del libro con el capítulo cinco, de nuevo analizando el caso de las enfermeras en Francia, pero esta vez centrándose en las estrategias de conciliación laboral y familiar. Moreno Colom escribe el capítulo ocho, que tiene por objetivo el análisis del tiempo de trabajo como factor de bienestar. Este capítulo relata las diferentes modulaciones que sufre el tiempo vital para los cuidadores (madres, hijas, etc.) de niños o ancianos. Un tiempo, el de los cuidadores, "constante, sin pausa ni porosidad alguna, sujeto a los ritmos" del cuidado (p.163). Finalmente el libro termina con un capítulo de Teresa Torns et al., centrado en las políticas del tiempo, originadas en el pensamiento de las feministas escandinavas y aplicado por primera vez en Italia en la década de los 90.

Clawson y Gerstel escriben el libro Unequal Times: Gender, Class and Family Employment Schedules, centrado en el caso de Estados Unidos, donde analizan las desigualdades de poder en el control sobre el tiempo por parte de los empleados sanitarios (doctores, enfermeras, técnicos y auxiliares). Estos investigadores se centran en examinar cómo se construyen las negociaciones en torno a los horarios laborales en los centros hospitalarios en base a dos variables clave a nivel individual, género y clase. Cercanos también a la sociología de las organizaciones, ponen el foco en cómo los tiempos laborales y familiares de los empleados están conectados: el movimiento de un empleado -ponerse enfermo, llegar tarde- afecta a la gestión del tiempo de sus compañeros de organización, y viceversa. El centrarse en las variables de género y clase les da la posibilidad de sacar a la luz cómo esos movimientos están condicionados por el poder otorgado por la posición ocupada en la estructura de clase y género. 
El diseño de investigación de Clawson y Gerstel es impecable. Con objeto de entender las desigualdades en el control sobre el tiempo de trabajadores, consideran tan solo cuatro ocupaciones del mismo sector: médicos, enfermeras, técnicos de hospital y auxiliares de enfermería. La elección de ocupaciones dentro del sistema sanitario para estudiar el control sobre el tiempo tiene una razón de ser: es una organización que funciona a turnos para estar operativa las 24 horas los 365 días del año. Como se observa, estas cuatro ocupaciones representan claramente ambas desigualdades, de género y de clase. Mientras que los médicos y los técnicos son profesiones masculinizadas, las enfermeras y las auxiliares son ocupaciones feminizadas. Asimismo, médicos y enfermeras representaran la clase media o media-alta, mientras que técnicos y auxiliares la clase trabajadora. La elección de este diseño así como la implementación de un análisis multimétodo y multinivel en la investigación es más que acertada: encuentran variación para la posible comparación pero dentro de un caso homogéneo, el sistema sanitario. Las variables a investigar (clase y genero) quedan representadas en sus distintas posiciones y eliminan el posible ruido introducido por sistemas laborales difícilmente comparables en sus lógicas temporales (por ejemplo, camareros con buzos). Sin embargo, aun homogeneizando por distintas ocupaciones dentro de un mismo sector, queda varianza no explicada. Dada la baja muestra (800 encuestas y 208 entrevistas), puede ser que las diferencias no analizadas dentro de una misma ocupación estén sesgando los resultados (por ejemplo, un cirujano y un médico de familia se enfrentan diariamente a condiciones temporales muy desiguales).

El libro Unfinished Revolution: Coming of Age in a New Era of Gender, Work, and Family de Kathleen Gerson, se centra en el análisis de los arreglos de empleo y cuidados en parejas de jóvenes entre 20 y 30 años en Estados Unidos: la primera generación nacida bajo la denominada revolución de género. Esta autora analiza bajo qué condiciones laborales y familiares se dan relaciones emocional y económicamente estables para dichas parejas. Así, realiza una descripción minuciosa sobre el origen de las expectativas y motivaciones laborales y familiares de los jóvenes, analizando a su vez las trayectorias de los padres de los entrevistados, y cómo estas están afectando a las prácticas y discursos de las parejas analizadas.

A pesar de una brillantez y claridad en la redacción de primer nivel, el diseño metodológico de Gerson es quizá el menos convincente. Parece claro que la longitudinalidad que Gerson ejecuta en el análisis de sus entrevistas cualitativas es más que atractiva. Se trata de diseñar tipologías de trayectorias vitales cruzando, por ejemplo, estructuras familiares y estados emocionales, y sus variaciones en el tiempo.
Algo así como un análisis de secuencias (Albott y Tsay, 2000) puramente cualitativo. Así, trata de identificar qué factores tienen en común las diferentes estructuras familiares -tradicionales, de doble ingreso o monoparentales- de cara a desarrollar hogares emocional y económicamente estables.

Resulta interesante la puesta en valor que Gerson realiza de los hitos vitales que producen cambios radicales en el curso de la vida de las familias analizadas. A lo largo del libro resulta como si la modificación de un factor vital (por ejemplo, una transición de desempleo al empleo por parte de una madre de familia) fuera la clave determinante del paso del malestar a la felicidad en la familia. Sin embargo, el ciclo de la vida proporciona tal riqueza (y complejidad) analítica, que en ciertos momentos se echa en falta la ilustración de otros factores: por ejemplo, no se tiene en cuenta cómo afecta en toda esta amalgama de estrategias familiares y de empleo, el efecto de la edad sobre el tiempo disponible y el bienestar emocional. En cualquier caso, solo localizarse en los hitos laborales y familiares ilustra una sencillez y contundencia expositiva que hacen de este libro un referente en los estudios de género, trabajo y familia.

\section{Poder y desigualdad en el CONTROL SOBRE EL TIEMPO}

En las últimas décadas, la mayoría de las investigaciones se han dedicado principalmente al estudio de las desigualdades de género, obviando la desigualdad de clase y la polarización social. Los trabajos de Martín-Criado y Prieto y de Clawson y Gerstel hacen una excelente contribución a los estudios del tiempo volviendo a enfatizar que las diferencias de género deben de analizarse siempre teniendo en cuenta los contextos de polarización socioeconómica. Esta quizá sea una de las principales cuestiones a echar en falta en el trabajo de Gerson. La variación introducida en el diseño de la autora neoyorkina es ciertamente escasa. Aunque tiene en cuenta individuos de diferentes grupos étnicos, clases sociales y procedencias territoriales, el grueso de sus entrevistados lo forman parejas jóvenes, de clase media, viviendo en Nueva York.

El enfoque cualitativo permite solventar vacíos en la investigación que es imposible analizar con datos de encuestas de uso del tiempo. Aquí un elemento de partida central considera que el control sobre el tiempo de cada individuo depende de su red de relaciones. Así como el tiempo disponible es un juego de suma cero (si te dedicas más al trabajo, tienes por obligación que quitar tiempo de la familia, o del ocio), el control del tiempo es dependiente. Cuando una persona, en su puesto de trabajo o su familia, toma una decisión que implica un uso temporal, está afectando sobre la gestión del tiempo de las personas de su entorno. Es decir, los tiempos 
de los individuos funcionan en cascada: de cómo uses tu tiempo dependerá el de tu entorno, y viceversa. Y allí donde hay dependencia (en este caso, temporal), hay poder. Mientras que los individuos de posiciones altas tienen elevados márgenes de decisión sobre el uso del tiempo, conforme más se desciende en la estructura social, más dependientes serán los usos del tiempo.

Esto es lo que Clawson y Gerstel han denominado "La red del tiempo". Martín Criado y Prieto parten del mismo punto. Mientras los primeros desarrollan este concepto basándose en un trabajo previo de Zerubavel (1979), los segundos utilizan el concepto de interdependencia desarrollado por Elias (1982). Como describen Martín Criado y Prieto al comienzo del libro: "lo que podamos hacer depende de las actividades de otras personas, la distribución de los tiempos de cada persona -y su poder de decisión sobre los mismos- depende siempre de los tiempos de las otras posiciones del entramado." (Pág. 7).

\section{EL EQUILIBRIO ENTRE TIEMPOS FAMILIARES Y LABORALES}

Los equilibrios temporales entre el empleo y la familia es un elemento central en la literatura de "workfamily balance" y las investigaciones aquí presentadas aportan nuevo conocimiento a dicha literatura. Es interesante remarcar cómo el tiempo de ambas esferas vitales es desigualmente percibido y protegido por los distintos estratos de clase.

En un seminario reciente donde se presentaba una investigación sobre usos del tiempo y familia en España, una persona del público espetó al ponente: "En tu investigación todo está mal porque partes de una hipótesis falsa: que las personas están deseando pasar tiempo con su familia (y que ese tiempo por definición es positivo)". La investigación que se presentaba era de excelente calidad. Pero quizá el comentario llevaba algo de razón. Esto es precisamente lo que encuentran Clawson y Gerstel en buena parte de sus entrevistados y también aparece en algunas de las entrevistas realizadas por Kathleen Gerson. Pero el rechazo al tiempo familiar no lo encuentran homogéneamente distribuido entre las distintas clases sociales: es una cuestión relativa a los individuos desaventajados.

Esta situación produce una paradoja. Según explican Clawson y Gerstel, las auxiliares de enfermería pasan muchas horas en su puesto de trabajo con objeto de pasar el menor tiempo posible con sus familias. El tiempo de trabajo aparece así como un refugio frente al tiempo familiar. Sin embargo, lo que encuentran estos investigadores es que las tensiones y problemas familiares vienen en buena medida originados por el estrés laboral y las largas jornadas de trabajo: es un círculo vicioso, y dañino, que afecta en mayor medida a las clases bajas. Este uso del tiempo de trabajo como refugio ante la familia ya fue descrito por Arlie Hochschild en su libro The Time Bind (1991).

También la investigación de Bouffartigue, incluida como capítulo en la compilación de Martín Criado y Prieto, encuentra resultados parecidos en una parte de su población estudiada: las enfermeras. Este autor identifica tres tipologías de dinámicas biográficas en las enfermeras investigadas, que denomina "todo por la profesión", "la enfermería como trabajo que aporta un complemento a los recursos de la familia" y "tensiones ligadas a la emancipación y a la conciliación". Tres tipologías que hacen eco a las controvertidas tipologías de la tesis de la preferencia de Catherine Hakim (2000) (mujeres orientadas al empleo, al hogar o ambivalentes). Sin embargo, Bouffartigue gira la relación causal y considera que en las mujeres que dejan de lado el tiempo familiar para dedicarse en plenitud al empleo -tipología denominada "todo por la profesión"-, lo hacen debido a las grandes exigencias laborales. Estas exigencias, dice el autor, "les llevarían al celibato" (pág. 129).

La cuestión central aquí, en realidad, es imposible de discernir: no podemos saber si el estrés y/o la fuerte orientación a lo laboral lleva a pasar menos tiempo en familia (o directamente no tenerla) o si se produce al revés. Es decir, no podemos definir con certeza la dirección de la causalidad con los datos actualmente disponibles.

\section{TIEMPOS FLEXIBLES}

Otro factor central que atraviesa el análisis del tiempo dedicado al empleo y la familia es la flexibilidad. Los estudios aquí presentados analizan la flexibilidad desde la crítica o la funcionalidad, dependiendo del autor. La funcionalidad predomina sobre la crítica en el conjunto de textos. Clawson y Gerstel distinguen entre dos tipos de flexibilidad. Por un lado, la "flexibilidad laboral", considerada como la capacidad de organizar el horario laboral en función de la demanda de trabajo percibida. Por otro lado, la "flexibilidad familiar", considerada como la capacidad para no afrontar presiones familiares para volver a casa a una hora concreta porque la pareja puede ocuparse del cuidado de la casa y de los niños.

Por su lado, para Gerson el concepto de flexibilidad se erige como el elemento central sobre el que se articula su investigación. Sin embargo, esta autora redefine el concepto de flexibilidad ampliándolo más allá de su concepción temporal. Cuando Gerson se refiere a la flexibilidad, no solo alude a la posibilidad de controlar la gestión del tiempo de trabajo, sino sobre todo a la posibilidad de intercambiar roles de género en una pareja. Es una flexibilidad de tiempo y género. Según esta autora, las parejas que logran adaptarse a situaciones sobrevenidas (por ejemplo, el desempleo del marido) adaptando sus roles de género (el marido desempleado pasa a ser el 
responsable principal de los cuidados) son aquellas parejas identificadas como poseedoras de una mayor estabilidad económica y, sobre todo, emocional. En palabras de la autora "la flexibilidad de género otorga a todos los tipos de familias una amplia variedad de opciones y recursos para responder a los retos. La capacidad y disposición a cruzar y diluir los límites entre la provisión económica y el cuidado de otros nos da la clave para entender por qué algunos hogares llegan a tener mayor capacidad de apoyo que otros" (pp. 44-45).

Sin embargo, la flexibilidad es desigualmente distribuida según clase y género. Esto es lo que discuten Clawson y Gerstel. Estos autores consideran que el género construye las demandas y deseos de un tipo particular de flexibilidad, mientras que la clase, en intersección con el género, forma la capacidad de disponer y ejecutar dicha flexibilidad. Los hogares de clases trabajadoras lo tienen difícil para controlar y gestionar su tiempo y acceder a horarios flexibles. Esta desventaja es doble para las mujeres de clases trabajadoras, que se enfrentan a una doble inflexibilidad: en el empleo y en la familia.

El cuidado de niños y el trabajo doméstico son las actividades que se llevan la mayor parte del tiempo no remunerado. Mientras que el tiempo dedicado al trabajo doméstico es más o menos flexible, la gran mayoría del tiempo de cuidado de niños es inflexible, el denominado en la literatura sobre cuidados a la infancia como "cuidado físico (o rutinario)": un cuidado que incluye actividades como cambiar pañales, dar de comer o acostar al niño y que cubre las necesidades básicas de éste. Un tipo de cuidado muy marcado por la desigualdad de género en el tiempo dedicado al mismo, además de ser el que, precisamente, más tiempo consume, como se observa en el siguiente gráfico para el caso de España:

Un acercamiento muy diferente a la flexibilidad realiza Martínez García en el primer capítulo de "Conflictos por el Tiempo". Este autor enfoca la fle- xibilidad desde la perspectiva de los empleadores, y no de los empleados. Mientras los empleados buscan flexibilidad para el correcto equilibrio vital, los empleadores buscan fuerza de trabajo flexible, esto es, siempre disponible. Aquí flexibilidad es utilizada retóricamente por empleadores para lo que otros autores como Standing (2011) Ilaman inestabilidad. Martínez García, en su análisis también de las enfermeras en el sector sanitario, considera un mercado laboral donde el mecanismo clave de valoración no son las competencias de los trabajadores sino sus disponibilidades temporales: es decir, su capacidad para tener tiempos flexibles y estar siempre disponibles para los empleadores.

Lo que Martín Criado y Carvajal en su análisis sobre el sindicalismo en las grandes superficies consideran como un tiempo donado. El mecanismo que ejecutarían los empleadores para una jerarquización laboral se basaría en la capacidad de sumisión temporal de los trabajadores: aquellos que más tiempo donan (a El Corte Inglés, a su hospital, etc.) ocuparían las posiciones de arriba en la jerarquía: "el buen empleado prolonga la jornada cuando se requiera, renuncia a las bajas a las que tiene derecho o pospone sus vacaciones; a cambio, obtiene recompensas salariales, permisos o cambios de turno cuando sus circunstancias familiares lo requieran" (Martín Criado y Carvajal, p. 53). Así, se establecería una lógica de dones y contra-dones, en términos maussianos (Mauss, 2009 [1925]). El tiempo aquí es la principal fuente de batalla entre jefes y trabajadores. Moreno Colom resalta que la lógica de la flexibilidad introduce nuevas lógicas de organización del trabajo invisibles para los trabajadores. Según esta autora, sería dicha invisibilidad la que explicaría que muchos de los jóvenes entrevistados en su investigación, a pesar de vivir con una incertidumbre temporal, éstos "viven positivamente la flexibilidad del tiempo de trabajo porque la perciben como una forma de libertad" (Moreno Colom, p. 150).

\section{Gráfico 1.}

Tiempo dedicado al cuidado de hijos. Padres activos de entre 25 y 55 años con al menos un menor de 13 años en el hogar (España, 2002 - 2010)
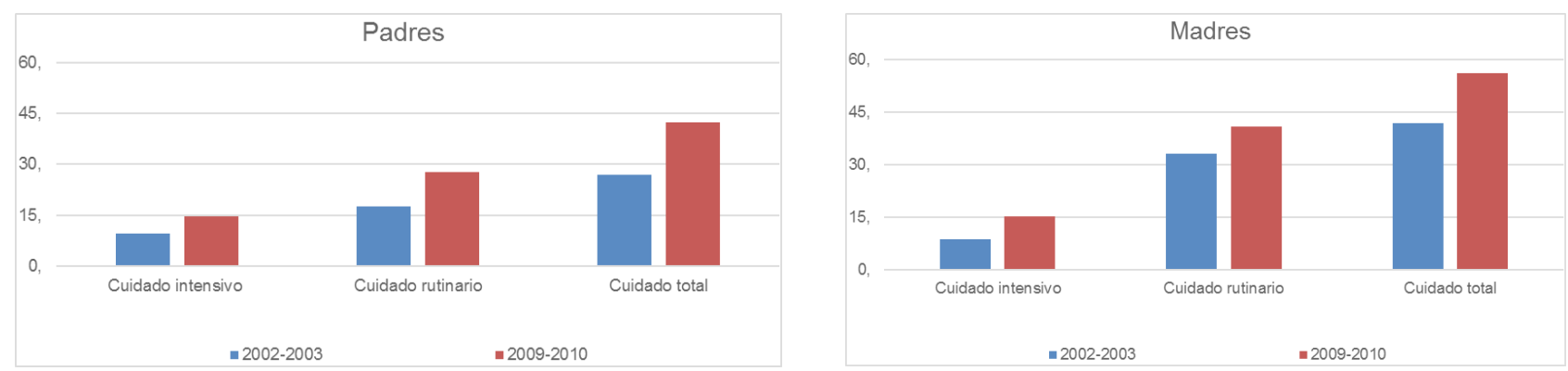

Elaboración propia a partir de las Encuestas de Empleo del Tiempo (2002-2003 y 2009-2010, Instituto Nacional de Estadística). Notas: Se grafican minutos diarios. Los valores son ponderados. $\mathrm{N}=5.481$ padres y 3,629 madres en EET 2002-2003 y N=2.061 padres y 1.681 madres en EET 2009-2010 
Lo que resalta Moreno Colom es la ambivalencia del uso del tiempo y la flexibilidad. Cuestión que es analizada por Clawson y Gerstel y Martínez García. La disponibilidad temporal y la flexibilidad son ambivalentes, desigualmente distribuidas entre las clases y desigualmente percibidas y/o recompensadas. Mientras que el hecho de poseer disponibilidad temporal para el empleo en los cuadros directivos es simbólicamente recompensada, en las mujeres de clases trabajadoras la disponibilidad temporal se da por hecha, para el empleo y para el hogar. Mientras que los hombres de clases altas utilizan su poder para poner más tiempo en el empleo, dada su recompensa simbólica por ello, las mujeres de clases altas utilizarían su poder para llevar más tiempo del empleo a la familia: así se reproduciría una vez más la masculinidad hegemónica (Connell, 2005), reforzando la cantidad de tiempo dedicada al empleo, que estaría positivamente asociada a la imagen de buen hombre proveedor; y la feminidad, asociada una vez más a la buena mujer cuidadora. Esto es lo que descubren Clawson y Gerstel, que terminan defendiendo que son los hombres de clases altas los que más están reproduciendo las desigualdades de género -por preferencia-, mientras que los hombres de clases bajas estarían deshaciendo ("undoing") el género -por constricción-.

\section{TIEMPO Y POLÍTICA SOCIAL}

Encontrar un equilibrio entre las políticas públicas y privadas que protejan los derechos y el tiempo de los trabajadores para cubrir las cada vez más complejas demandas de cuidados parece ser clave de cara a la productividad de los empleados y la fortaleza de las familias. Sin embargo, en España no parece que el camino tomado por el Estado esté surtiendo el efecto deseado.

Las políticas desarrolladas para proteger el tiempo de los trabajadores, no solo son insuficientes sino que, terminan generando más desigualdad, apuntan Clawson y Gerstel. Como es sabido, dichas políticas se han desarrollado de forma precaria en Estados Unidos, donde ni tan siquiera existe ningún tipo de permiso de paternidad. Pero las existentes, como la Family and Medical Leave Act (FMLA) de 1993, terminan generando dinámicas desigualitarias para los trabajadores: mientras las enfermeras bien posicionadas hacen uso de estas políticas, las auxiliares ni siquiera aparecen como poseedoras de la información necesaria para hacer un correcto uso de las prestaciones sociales de tiempo.

Diferentes investigaciones han encontrado resultados similares para el caso de España. Serrano et al. (2014) analizaron el potencial desigualitario de la Ley de Dependencia de 2006. Esta ley, que se desarrolló para proclamar el derecho universal a ser cuidado por parte de los dependientes, así como para proteger y dignificar el tiempo de los cuidadores, terminó reproduciendo desigualdades de clase y género (Serrano et al., 2014; Cano, 2014). Similares resultados encontró Lapuerta (2012) para los permisos parentales en España. Los resultados de estas investigaciones recientes dejan clara la necesidad de una reevaluación de las políticas sociales destinadas a proteger el tiempo familiar de los trabajadores. Iniciativas como la PPIINA (Plataforma por los Permisos Iguales e Intransferibles de Nacimiento y Adopción) reclaman con fuerza la igualación entre hombres y mujeres de los tiempos dedicados a nuestros hijos.

La compilación de Martín Criado y Prieto termina con un capítulo dedicado a las políticas del tiempo (Torns et al., pp. 169-190). Las políticas del tiempo planteadas originariamente por científicas sociales escandinavas y aplicadas por primera vez en Italia en 1990, tenían como objetivo el desarrollo de iniciativas políticas de protección del tiempo con perspectiva de género. Sin embargo, los valiosos intentos para el desarrollo de políticas sociales del tiempo en España (Ley de "Dependencia" -2006, de "Igualdad" -2007- o de "Conciliación" -1999-) han devenido en eso, intentos. El punto de partida a finales de los noventa y principio de los dos mil era optimista: era el comienzo de las políticas de protección del tiempo. Estas se irían poniendo en práctica y progresivamente mejorando sus debilidades, como los efectos no intencionados mencionados más arriba, y aumentando su financiación y cobertura. Nada más lejos de la realidad: la llegada de la Gran Recesión en 2007 generó un escenario desolador, siendo España uno de los países más golpeados del mundo y terminando por ralentizar, si no revertir, este gran proyecto. Desde entonces, el tiempo ha vuelto a convertirse en lo que nunca dejó de ser: oro.

\section{A MODO DE CONCLUSIÓN}

El tiempo es una cuestión central de la vida cotidiana. Los cambios sociodemográficos de las últimas décadas han modificado sin precedente nuestros usos del tiempo: el hecho de que la gente viva más tiempo, la incorporación de las mujeres al mercado de trabajo o el aumento sin cesar del ocio han alterado nuestra disponibilidad temporal. Las batallas por el tiempo entre empleadores y empleados o entre distintas clases sociales tienen consecuencias determinantes no solo para los que libran dichas batallas sino para los que nunca eligieron librarlas: los niños. Recordemos que la futura estabilidad emocional y económica de los niños de hoy depende en buena medida de la cantidad y la calidad de tiempo parental que estén recibiendo (ver, por ejemplo, Fiorini y Keane, 2014). Por tanto, de las actuales luchas por el tiempo dependerá el futuro de nuestras sociedades. 
Leyendo las investigaciones aquí señaladas observamos con claridad la relevancia teórica y empírica de la cuestión temporal. Los autores ponen de manifiesto dos cuestiones en los estudios de uso del tiempo. Por un lado, la necesidad de centrarse en las estructuras organizacionales. Por otro, la vuelta al análisis desde la clase social. Tres grandes investigaciones que recomiendo a todos aquellos investigadores interesados en el análisis de clase, género y, por supuesto, tiempo.

\section{Notas}

$1 \quad$ Este trabajo ha contado con la ayuda de los proyectos CSO2012-33476 y CSO2014-58754-P así como la ayuda para la formación de doctores BES-2013066777, financiados por el Ministerio de Economía y Competitividad.

\section{REFERENCIAS BIBLIOGRÁFICAS}

Albott, A. y Tsay, A. 2000. "Sequence Analysis and Optimal Matching Methods in Sociology". Sociological Methods and Research 29 (1): 3-33. https://doi.org/10.1177/004 9124100029001001

Cano, T. 2014. "Los cuidados en conflicto. De la Ley de Dependencia a las estrategias de cuidados familiares". Tesis de Máster, Departamento de Sociología IV, Universidad Complutense de Madrid, Madrid.

Castell, M. 2009. Communication Power. Oxford University Press: New York.

Clawson, D. and Gerstel, N. 2014. Unequal Time: Gender, Class and Family Employment Schedules. New York: Russell Fundation.

Connell, R. W. (2005): Masculinities. Berkeley: University of California Press.

Elias, N. 1982. Sociología Fundamental. Barcelona: Gedisa.

España. 1999. "Ley 39/1999, de 5 de noviembre, para promover la conciliación de la vida familiar y laboral de las personas trabajadoras". Boletín Oficial del Estado 266: 38934-38942, 6 de Noviembre de 1999.

España. 2006. "Ley 39/2006, de 14 de diciembre, de Promoción de la Autonomía Personal y Atención a las personas en situación de dependencia". Boletín Oficial del Estado 299: 44142 a 44156, 15 de Diciembre del 2006.

España. 2007. "Ley Orgánica 3/2007 de 22 de marzo, para la igualdad efectiva de mujeres y hombres". Boletín Oficial del Estado 71: 12611 a 12645, 23 de Marzo de 2007.

Fiorini, M. y Keane, M. 2014. "How the allocation of children's time affects cognitive and noncognitive development".
Journal of Labour Economics 32(4): 787-834. https:/l doi.org/10.1086/677232

Gerson, K. 2011. The Unfinished Revolution: Coming of Age in a New Era of Gender, Work and Family. Oxford: Oxford University Press.

Hochschild, A. 1997. The Time Bind. When Work Becomes Home and Home Becomes Work. Holt Paperbacks: New York.

Hakim, C. 2000. Work-lifestyle Choices in the 21 $1^{\text {st }}$ Century: Preference Theory. Oxford: Oxford University Press.

Lapuerta, I. 2011. Employment, Motherhood and Parental Leaves in Spain. Tesis Doctoral, Universidad Pompeu Fabra: Barcelona.

Martín Criado, E. y Prieto, C. comp. 2015. Conflictos por el tiempo: Poder, relación salarial y relaciones de género. Madrid: UCM/CIS.

Mauss, M. 2009. Ensayo sobre el don. Forma y función del intercambio en las sociedades arcaicas. Katz Editores: Buenos Aires.

Serrano, A. et al. 2014. "Crisis de los cuidados, Ley de Dependencia y confusión semántica". Revista Internacional de Sociología 71(3): 669-674. https://doi.org/10.3989/ ris.2012.07.30

Standing, G. 2011. The Precariat. The New Dangerous Class. Bloomsbury: London.

Zerubavel, E. 1979. Patterns of Time in Hospital Life: A Sociological Perspective. The University of Chicago Press: Chicago.

TOMÁS CANO. Doctorando en la Universitat Pompeu Fabra e investigador FPI en la Universitat Autónoma de Barcelona. Anteriormente ha sido investigador contratado en la UCM. Licenciado en Sociología (UGR, 2010) y Máster en Metodología de la Investigación por la UCM (2012), ha trabajado y colaborado en diferentes proyectos I+D nacionales e internacionales. Actualmente realiza su tesis doctoral sobre inversiones temporales parentales al cuidado infantil y el desarrollo cognitivo de los hijos, usando datos de uso del tiempo longitudinales y transversales. Ha realizado estancias de investigación en la University College of London (UK) y en la University of Queensland (Australia), donde es research fellow del ARC Centre of Excellence for Children and Families over the Life Course. En 2013 fue galardonado con el "Premio Jóvenes Sociólogos" que otorga la Asociación Madrileña de Sociología. Sus áreas de investigación principal se centran en la metodología de investigación, dinámicas de género y familia, desigualdad, desarrollo infantil y políticas sociales. 